\title{
Review of Serum Urea-Albumin Ratio as a Prognostic Marker in Critical Patients with Non-Chronic Kidney Disease (non-CKD)
}

\author{
Deepak Gundpatil • B. L. Somani
}

Published online: 14 June 2014

(C) Association of Clinical Biochemists of India 2014

\section{Dear Editor,}

We thank readers for their comments on the article "Serum Urea-Albumin Ratio as a prognostic marker in critical patients with non-Chronic Kidney Disease (non-CKD)".

We agree that Urea-Albumin ratio (UAR) is a surrogate marker for evaluating prognosis in Non-CKD patients and therefore there is bound to be some element of positive or negative bias depending on individual patient's clinical condition and therapeutic profile. However, as mentioned by reader that urea could be affected in patients on tetracycline therapy but what has been reported is that it happens in renal disease patients because of increase in halflife of tetracycline which reaches to toxic levels [1]. However, as reported in the article these cases were excluded from the study. As regards the comment that dehydration and infection affecting Urea levels, we feel that the significance of ratio would not be affected in ICU patients as presence of infection would obviously affect the prognosis. As regards dehydration, UAR would not be significantly affected, as both urea and albumin levels would have increased, minimally affecting the UAR.

Moreover, in the present study we have taken patients with wide range of different clinical diagnosis (50\% Surgical \& $50 \%$ Medical ICU Patients, respectively) excluding the patients with conditions which will affect urea or albumin parameters by clinical history such as
Chronic Kidney or Liver disease and intestinal malabsorption, etc [2].

Also, we have considered the parameters in blood sample collected on first day of admission so that the Urea or Albumin levels would not be affected due to subsequent treatment in ICU stay. Considering this, the drugs or treatment during stay in ICU shouldn't cause any change in our findings.

Considering the sensitivity of $67.1 \%$ and specificity of $83 \%$ at a cut-off of 23.44 UAR, it is a cost effective prognostic marker and can be an important guide in attending to ICU patients with critical evaluation and extensive treatment for an improved out come. However, we do agree that UAR is a surrogate marker for Prognosis in ICU patient and there is always a scope to find new prognostic marker with better predictive value.

\section{References}

1. Munar M, Singh H. Drug dosing adjustments in patients with chronic kidney disease. Am Fam Physician. 2007;75(10):1487-96.

2. Gundpatil DB, Somani BL, Saha TK, Banerjee M. Serum urea: albumin ratio as a prognostic marker in critical patients with nonchronic kidney disease. Indian J Clin Biochem. 2014;29(1): 97-100. 